\title{
FACIAL FEATURE EXTRACTION TECHNIQUES FOR FACE RECOGNITION
}

\author{
Rahib H. Abiyev \\ Applied Artificial Intelligence Research Centre, Near East University, Nicosia, North Cyprus, Mersin-10, Turkey
}

Received 2014-03-06; Revised 2014-03-18; Accepted 2014-10-09

\begin{abstract}
Face recognition is one of the biometric techniques used for identification of humans. The design of the face recognition system includes two basic steps. The first step is the extraction of the image's features and the second one is the classification of patterns. Feature extracting is a very important step in face recognition. The recognition rate of the system depends on the meaningful data extracted from the face image. If the features belong to the different classes and the distance between these classes are bigger then these features are important for recognition of the images. In this study, the design of face recognition system using three different feature extraction techniques- Principal Component Analysis (PCA), Fisher Linear Discriminant Analysis (FLD) and Fast Pixel Based Matching (FPBM) is presented. The comparative analysis of the simulation results of these methods is presented.
\end{abstract}

Keywords: Face Recognition, PCA, FLD, Fast Pixel Based Matching

\section{INTRODUCTION}

Since the last century biometric techniques were used for identification of humans. Faces are one of many forms of biometrics used to identify individuals and to verify their identity. Face recognition refers to the automated method of verifying a match between two human faces. Feature extraction is a very important step in face recognition. The recognition rate of system depends on the meaningful data extracted from the face image. If the features belong to different classes and the distance between these classes is large then these features are important for a given image. There is no $100 \%$ matching between the images of the same face even if they were from the same person. In this study, the analysis of face recognition systems using three different feature extraction techniques-Principal Component Analysis (PCA), Fisher Linear Discriminant analysis (FLD) and Fast Pixel Based Matching (FPBM) is considered.

PCA is a technique that takes high-dimensional image data and uses the dependencies between the variables to represent it in a more tractable, lower- dimensional form, without losing too much information. PCA is a statistical procedure that evaluates the covariance structure of a set of variables and identifies the principal directions in data variables. PCA is used to identify sets of orthogonal coordinate axes through the data. Principal components are determined by computing eigenvectors and eigenvalues of the data covariance matrix. Based on principal components the identification of face images is performed.

FLD is the most famous way to search for trends in the data, which has the largest difference and highlight data. This method is also used, for lower-dimensional representation of the data, which removes some of the trends "noisy". The basic idea of FLDA is the design an optimal transform, which can maximize the ratio of between-class to within-class scatter matrices so that the classes can be well separated in the low-dimensional space. FLD method allows information between members of the same category (images of the same person) to develop a set of feature vectors. FLD uses a linear projection of the $n$-dimensional data onto a one-dimensional space (i.e., line). Projection onto a line is separated by a class and classification problem becomes choosing a line. 
FPBM is a method to extract the features of the images on the basis of matching image areas and subpixel displacement estimate using similarity measures. The recognition is based on the edge detection. This method generates much less information than the original image has. This is because it eliminates most of the details that are not relevant for the purpose of identifying the boundaries, while preserving the essential information to describe the shape and structural characteristics and geometry of the objects represented.

This study describes the design of a face recognition system using PCA, FLD and FPBM methods. Each of these techniques was implemented in MATLAB. The outputs of the feature extraction block are classified to recognize the face patterns. The algorithm uses Euclidean Distance for classification of face images. Comparisons of the simulation results of face recognition systems using PCA, FLD and FPBM algorithms are presented.

\section{FEATURE EXTRACTION TECHNIQUES}

\subsection{Principal Component Analysis}

In the result of application of the PCA algorithm an original data of image is projected into a new coordinate space. Each coordinate axis in the new coordinate space will represent a principal component vector. The first principal component vector is the direction along which the variance is a maximum; the second principal component vector is defined by the direction which maximizes the variance among all directions orthogonal to the first vector and so on. PCA algorithm includes the following steps (Smith, 2002; Wold et al., 1987; Tzimiropoulos et al., 2011).

The first step is the reading of the face images from the database and converting them into grayscale values. After these operations obtained $2 D$ face images are converted into $1 D$ image vector. The images are converted to represent each face image of dimensions $N \mathrm{x} N$ to single beam of dimensions $N \times N$ to single beam of dimensions $N^{2} x 1$. The data are stored in the $T=[T \alpha]$ vector. Here $\alpha$ is the converted image represented in $1 D$, $T$ is the vector that contains all converted images.

In the second step the mean of images of $T$ vector is calculated Equation 1:

$$
m=\frac{1}{X} \sum_{i=1}^{X} T_{i}, i=1,2, \ldots, X
$$

where, $m$ is a mean, $X$ is a number of images in the database.

In the third step the deviation $\Phi_{i}$ of each image from the mean image are determined Equation 2:

$\Phi_{i}=T_{i}-m, i=1,2, \ldots, X$

In the fourth step the eigenvectors of the covariance matrix $C=A \times A^{T}$ are calculated. Here $A=\left[\Phi_{1}, \Phi_{2}, \ldots\right.$, $\left.\Phi_{X}\right]$. In this step it is necessary to solve the eigenvalue problem (Turk and Pentland, 1991) Equation 3:

$$
C U=U \Lambda
$$

Here $\Lambda$ is a diagonal matrix that represents the eigenvalues of the matrix $C$ and $\Lambda=\operatorname{diag}\left[\lambda_{1}, \lambda_{2}, \ldots\right.$, $\lambda_{N N}$ ]. $U$ is the associated eigenvectors of $\lambda$. These eigenvectors represent the new face space.

In the fifth step a centred image vector is projected into face space Equation 4:

$$
\begin{aligned}
& \text { temp }=U^{T} A \\
& P=[\text { Ptemp }]
\end{aligned}
$$

where, $P$ is a vector that contains all projected images. The original image vector A may be reconstructed from the projections:

In the sixth step PCA features are extracted from the test images

In the seventh step Euclidean distances are calculated (Saito and Toriwaki, 1994; Danielsson, 1980) Equation 5:

$$
\text { temp }=[\operatorname{norm}(\breve{P}-P(i))]^{2}, E=[\text { Etemp }]
$$

where, $E$ is the Euclidean distance vector.

In the eighth step the minimum Euclidean distance using $\min (x)$ function is computed. The corresponding index with the minimum distance is the recognized image.

\subsection{Fisher Linear Discriminant Analysis}

Using FLD algorithm the calculations of the "within scatter matrix" and the "between scatter matrix" are performed to obtain the projected fisher images that are used in recognition (Welling, 2005; Tucker et al., 1997; Mika et al., 1999). Fisher's linear discriminant function J is defined using covariance matrices. FLD considers maximizing the following objective Equation 6:

$$
J(w)=\frac{W^{T} S_{B} W}{W^{T} S_{W} W}
$$


Where:

- $S_{B}$ is the "between classes scatter matrix" and

- $S_{W}$ is the "within classes scatter matrix"

They are defined as Equation 7 and 8:

$$
\begin{aligned}
& S_{B}=\sum_{C} N_{C}\left(\mu_{C}-\bar{X}\right)\left(\mu_{C}-\bar{X}\right)^{T} \\
& S_{W}=\sum_{C} \sum_{i \in C}\left(X_{i}-\mu_{C}\right)\left(X_{i}-\mu_{C}\right)^{T}
\end{aligned}
$$

Where Equation 9 and 10:

$$
\begin{aligned}
& \mu_{C}=\frac{1}{N_{C}} \sum_{i \in C} X_{i} \\
& \bar{X}=\frac{1}{N_{C}} \sum_{i} X_{i}=\frac{1}{N_{C}} \sum_{C} N_{C} \mu_{C}
\end{aligned}
$$

where, $N_{C}$ is a number of cases in the class $C$ Equation 11:

The total scatter $S_{T}$ :

$$
S_{T}=\sum_{i}\left(X_{i}-\bar{X}\right)\left(X_{i}-\bar{X}\right)^{T}
$$

Is given by Equation 12:

$$
S_{T}=S_{W}+S_{B}
$$

The next step is the eliminating of eigenvalues and sorting all non-zero eigenvalues in descending order as $V$, where $V$ is FLD eigenvector. The computing of the projected images using FLD algorithm is performed as Equation 13:

$$
\bar{P}=V^{T} \times P
$$

In next step FLD features are extracted from the test image. Then Euclidean distances are calculated as (Saito and Toriwaki, 1994; Danielsson, 1980) Equation 14:

$$
\text { temp }=[\operatorname{norm}(\breve{P}-P(i))]^{2}, E=[\text { etemp }]
$$

where, $E$ is the Euclidean distance vector.

Finally the minimum Euclidean distance using min (x) function is computed. The corresponding index with the minimum distance is the recognized image from database folder.

\subsection{Fast Pixel Based Matching using Edge Detection}

The recognition of the contours (edge detection) is used for the purpose of marking the points of a digital image in which the light intensity changes abruptly. Abrupt changes of the properties of an image are usually a symptom of events or major changes of the physical world. These changes can be discontinuity of the depth in the surface, changing the properties of materials and variations in lighting conditions from the surrounding environment. The edge detection is a research field of image processing, particularly the branch of feature recognition. The operation of edge detection generates images containing much less information than the original, because it eliminates most of the details that are not relevant for the purpose of identifying the boundaries (Marr and Hildreth, 1980; Fraser, 1985).

The methods used for edge detections can be grouped into two categories: Search-based and zero-crossing based. Search based methods recognize the contours by computing the maxima and the minima of the first order derivative of the image, usually looking in the direction in which we have the maximum local gradient. Zero-crossing based methods seek for the zero-crossing points at which the derivative of the second order passes through zero, usually the Laplacian function or a differential expression of a non-linear function.

The contours play a very important role in many applications of computer vision. A typical contour could be, for example, the boundary between an area of red colour and a yellow, or a line with a thickness of a few pixels and a different colour compared to a uniform colour background.

The model illustrated here, has a function error erf that can be used to create a mathematical model of the effects of the blurs sufficient accurate to describe many practical applications. An image $f$ with a onedimensional contour positioned exactly in 0 can be represented then by the following function Equation 15:

$$
f(x)=\frac{I_{r}-I_{l}}{2}\left(\operatorname{erf}\left(\frac{x}{\sqrt{2 \sigma}}\right)+1\right)+I_{l}
$$

\subsubsection{Calculation of the First Derivative}

Many algorithms for the recognition of contours operate on the first order derivative of the light intensitywhich corresponds to the gradient of the intensity of the initial image. Based on this we search the peak values of 
the gradient of intensity. If $I(x)$ represent the intensity of pixel $x$ and $I^{\prime}(x)$ denotes the derivative (gradient intensity) to the pixel $x$, we get Equation 16:

$$
I^{\prime}(x)=-1 / 2 \cdot I(x-1)+0 . I(x)+1 / 2 \cdot I(x+1)
$$

\subsubsection{Calculation of the Second Derivative}

Other operator for edge detection is based on a calculation of the second order derivative of the intensity, which roughly corresponds to the rate of change of the gradient. In the ideal case-in which the intensity varies in a continuous manner-the second derivative vanishes at the points of maximum gradient. This method, however, works well only if the image is represented in a suitable scale. As explained before, a line corresponds to a double contour and then you will have a gradient of intensity on one side of the line, immediately followed by a gradient of opposite value on the opposite side. For this reason it can be expected to have large variations in the gradient images containing lines.

If $I(x)$ is the intensity value at the point $x$ and $I$ ' $(x)$ is the second derivative at the point $x$, then the following relation holds Equation 17:

$$
I^{\prime \prime}(x)=1 . I(x-1)-2 . I(x)+1 . I(x+1)
$$

Nowadays set of operators is applied for edge detection operation. Roberts, Prewitt, Sobel operators of the first order, Marr-Hildreth method based on the second order. Currently the Canny algorithms-and its variants - are the most used method for the recognition of contours. None of the numerous other subsequently proposed methods have so far proved more effective, except in very specific applications. In his original work, Canny set out to find a filter that would eliminate the noise in the image. The filter could be well approximated by a Gaussian kernel of the first order. Canny also introduced the concept of non-maximum suppression, in which the gradient reaches the maximum value in the estimated direction of the gradient. The search for non-maximum in a grid of points can be implemented by calculating the gradient direction with the first derivative, rounding the direction found in multiples of $45^{\circ}$ and finally compared with the values of amplitude of the gradient in the direction calculated.

\section{SIMULATION RESULTS}

The simulation is performed on the ORL face database which contains a set of face images taken between April 1992 and April 1994 at the Olivetti
Research Laboratory in Cambridge University (http://www.cl.cam.ac.uk/research/dtg/attarchive/pub/dat a/att_faces.zip). For some persons, the images were taken at different times, varying the lighting, facial expressions- open, closed eyes, smiling, not smiling and facial details- glasses and no glasses. Fig. 1 depicts samples of original images used for recognition.

The simulation of the facial recognition system is performed using PCA, FLD and FBPM feature extraction techniques. Some of these methods have been analysied in (Chan et al., 2010). In this study we are unified these three feature extraction methods for dace recognition and give their comparative results. The classifications of the face images are performed by measuring Euclidian distance. The simulations are performed in two stages. On the first stage the noisy images (Fig. 2), in the second stage the face images without noisy are considered for recognition (Fig. 3).

In the first experiment, 138 images of 23 persons are taken. In the database each person has 6 different images. In this experiment the number of test noisy images is 46 (Table 1). Using PCA the 40 images were recognized successfully and recognition rate (R.R) was $86.9 \%$. Using FLD the 42 images were recognized successfully and recognition rate was $91.3 \%$. Using FPBM the 36 images were recognized successfully and recognition rate was $78.2 \%$.

In the second experiment, 231 images are taken. In the database these images belong to 33 persons. Each person has 7 different images. Number of noisy test images used in this experiment was 99. Using PCA the 91 images were recognized successfully with recognition accuracy $91.9 \%$. Using FLD the 92 images were recognized successfully with recognition accuracy 92.9\%. Using FPBM the 75 images were recognized successfully with recognition accuracy $75.6 \%$.

In the third experiment, 320 images are taken. These images are belong to 40 persons and each person has 8 different images. A number of noisy test images used in this experiment were 80 . Using PCA the 71 images were recognized successfully with recognition accuracy $88.7 \%$. Using FLD the 75 images were recognized successfully with recognition accuracy $93.8 \%$. Using FPBM the 59 images were recognized successfully with recognition accuracy $73.8 \%$.

In the next stage the simulation results were obtained using non-noisy test images from the second set. Table 2 shows the simulation results of non-noisy face recognition system using PCA FLD and FPBM algorithms. 
In the first experiment, 65 images of 13 persons are taken. In this image database each person has 5 different images. Number of test images that are used in this experiment is 65. Using PCA the 59 images were recognized successfully with the recognition rate $90.8 \%$. Using FLD the 62 images were recognized successfully with the recognition rate $95.4 \%$. Using FPBM the 45 images were recognized successfully with the recognition rate $69.2 \%$.
In the second experiment, 135 images of 27 persons are taken. Each person has 5 different images. Using PCA the 127 images were recognized successfully with recognition accuracy $94.1 \%$. Using FLD the 130 images were recognized successfully with recognition accuracy $96.2 \%$. Using FPBM the 90 images were recognized successfully with recognition accuracy $66.7 \%$.

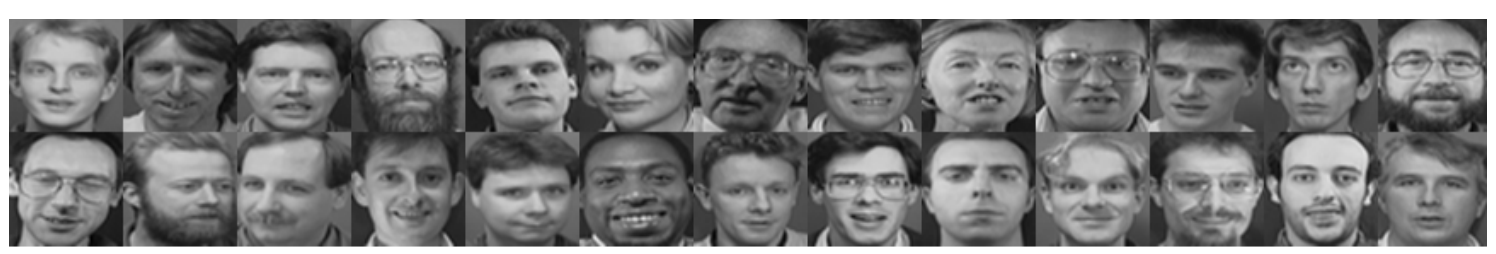

Fig. 1. Samples of original images

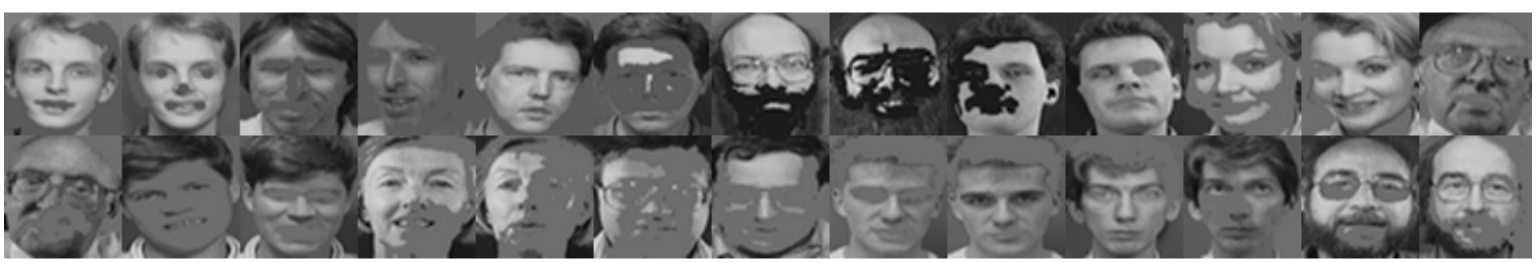

Fig. 2. Noisy test images

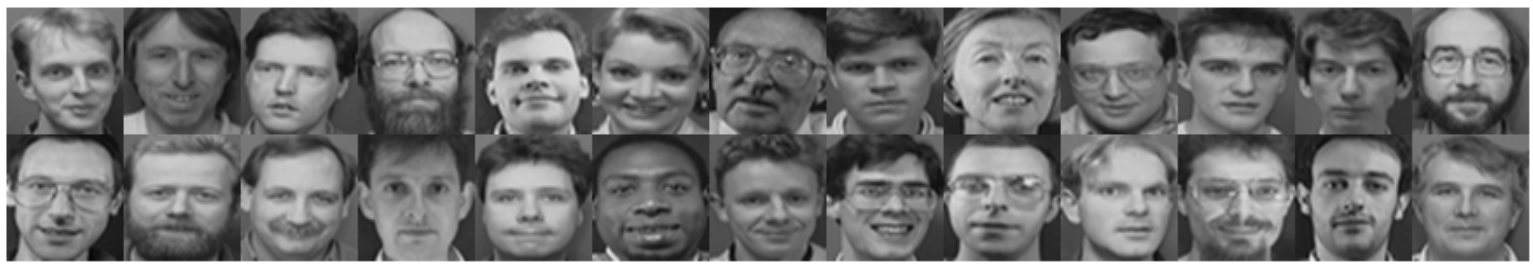

Fig. 3. Test images without noisy

Table 1. Recognition rates of the system for noisy tested face images

\begin{tabular}{llllll}
\hline Subjects & Tested faces & Faces in database & PCA R.R $(\%)$ & FLD R.R $(\%)$ & FPBM R.R $(\%)$ \\
\hline 23 & 46 & 138 & 86.90 & 91.30 & 78.20 \\
33 & 99 & 231 & 91.90 & 92.90 & 75.60 \\
40 & 80 & 320 & 88.70 & 93.80 & 73.80 \\
\hline
\end{tabular}

Table 2. Recognition rates of the system for non-noisy tested face images

\begin{tabular}{llllll}
\hline Subjects & Tested faces & Faces in database & PCA R.R $(\%)$ & FLD R.R $(\%)$ & FPBM R.R $(\%)$ \\
\hline 13 & 65 & 65 & 90.80 & 95.40 & 69.20 \\
27 & 135 & 135 & 94.10 & 96.20 & 66.70 \\
40 & 200 & 200 & 94.50 & 95.50 & 62.50 \\
\hline
\end{tabular}


In the third experiment, 200 images are taken. These images belong to 40 persons and each person has 5 different images. Using PCA the 189 images were recognized successfully with recognition accuracy 94.5\%. Using FLD the 191 images were recognized successfully with recognition accuracy $95.5 \%$. Using FPBM the 125 images were recognized successfully with recognition accuracy $62.5 \%$. The simulation results demonstrate the efficiency of using of PCA and FLD methods over FPBM method in face recognition.

\section{CONCLUSION}

Three different feature extraction methods (PCA, FLD and FPBM) are applied for face recognition. Euclidean distance is used for classification of the face images. The structure of the face recognition system was designed and the computer simulation of the recognition system has been performed for two kinds of face images: Noisy and without noisy cases. The simulations have been done using different number of images. The best average recognition rate for noisy and non-noisy images was obtained using FLD method. The recognition rate of 40 images was obtained using FLD method equals to $93.8 \%$ and $95.5 \%$ for noisy and non-nosy cases respectively. The performances of the FPBM for the given images were $73.8 \%$ and $62.5 \%$ respectively. The performances of the PCA for the given images were $88.7 \%$ and $94.5 \%$ respectively. The simulation results demonstrate that the FLD method is useful for recognition of face images.

\section{REFERENCES}

Chan, L.H., S.H. Salleh and C.M. Ting, 2010. Face biometrics based on principal component analysis and linear discriminant analysis. J. Comput. Sci., 6: 693-699. DOI: 10.3844/jcssp.2010.693.699

Danielsson, P.E., 1980. Euclidean distance mapping. Comput. Graphics Image Proc., 14: 227-248.

Fraser, C.M., 1985. Calculation of higher derivative terms in the one-loop effective Lagrangian. Zeitschrift für Physik C Particles and Fields, 28: 101-106. DOI: 10.1007/BF01550255
Marr, D. and E. Hildreth, 1980. Theory of edge detection. Proc. Royal Soc. London. Series B., 207: 187-217. DOI: $10.1098 / \mathrm{rspb} .1980 .0020$

Mika, S., G. Ratsch, J. Weston, B. Scholkopf and K.R. Mullers, 1999. Fisher discriminant analysis with kernels. Processing of the IEEE Signal Processing Society Workshop Neural Networks for Signal Processing IX, (SPSW, 99), IEEE Xplore Press, Madison, WI, pp: 41-48. DOI: 10.1109/NNSP.1999.788121

Saito, T. and J.I. Toriwaki, 1994. New algorithms for euclidean distance transformation of an-dimensional digitized picture with applications. Patt. Recognit., 27: 1551-1565. DOI: 10.1016/00313203(94)90133-3

Smith, L.I., 2002. A tutorial on principal components analysis. Cornell University, USA.

Tucker, K., S.P. Rushton, R.A. Sanderson, E.B. Martin and J. Blaiklock, 1997. Modelling bird distributionsa combined GIS and Bayesian rule-based approach. Landscape Ecol., 12: 77-93. DOI: 10.1007/BF02698209

Turk, M.A. and A.P. Pentland, 1991. Eigenfaces for Recognition. J. Cognitive Neuroscience, 3: 71-86. DOI: 10.1162/jocn.1991.3.1.71

Tzimiropoulos, G., S. Zafeiriou and M. Pantic, 2011. Principal component analysis of image gradient orientations for face recognition. Proceedings of the IEEE International Conference on Automatic Face and Gesture Recognition and Workshops, Mar. 2125, Santa Barabara, USA. pp: 553-558. DOI: 10.1109/FG.2011.5771457

Welling, M., 2005. Fisher linear discriminant analysis. Department of Computer Science, University of Toronto.

Wold, S., K. Esbensen and P. Geladi, 1987. Principal component analysis. Chemometrics Intelligent Laboratory Syst., 2: 37-52. DOI: 10.1016/01697439(87)80084-9 